\title{
Inhalative Kortikoide gegen COPD machen die Knochen brüchig
}

Die langzeitige Therapie chronisch obstruktiver Lungenerkrankungen (COPD) mit inhalativen Glukokortikoiden wirkt sich negativ auf die Stabilität des Knochengerüsts aus. Zwar steigt das Frakturrisiko nur um circa $27 \%$. Doch der Unterschied ist signifikant und bezeichnet angesichts der großen Zahl gefährdeter Patienten ein bedeutendes klinisches Problem.

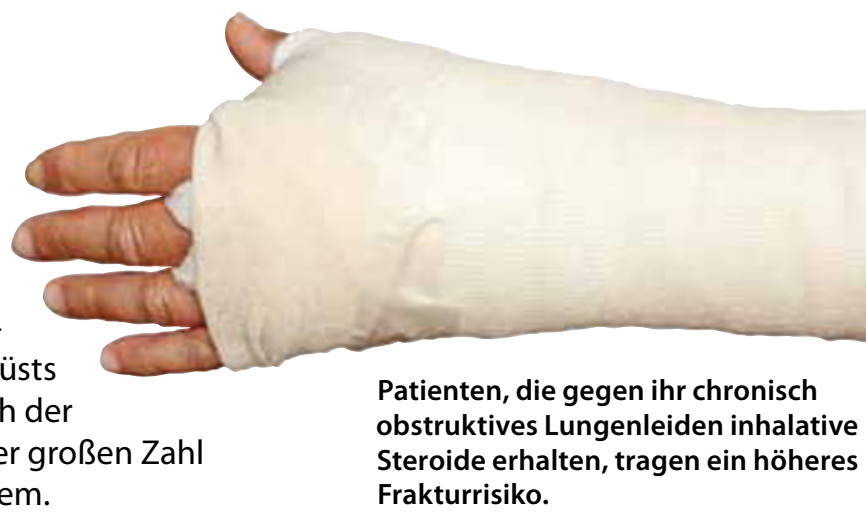

[ür ihre Studie hatten amerikanische und britische Mediziner 16 randomisierte und kontrollierte Studien sowie sieben Beobachtungsstudien metaanalysiert. Insgesamt wurden darin 9.143 COPD-Patienten, die mit den inhalativen Kortikosteroiden (IKS) Fluticason oder Budesonid behandelt wurden, mit 8.370 Kontrollpatienten verglichen, die gegen ihre chronischen Lungenleiden keine Kortikoide erhielten. Die Dauer der IKS-Behandlung betrug jeweils mindestens 24 Wochen.

Es zeigte sich, dass IKS das Frakturrisiko in den kontrollierten Studien signifikant erhöhten (Odds Ratio [OR] 1,27; $\mathrm{p}=0,04)$. Aus den Daten der Beobachtungsstudien errechneten die Wissenschaftler ebenfalls eine höhere Frakturrate unter IKS (OR 1,21; p < 0,001), wobei ein Anstieg der Dosis um ein Beclomethason-Äquivalent von $500 \mu \mathrm{g}$ gleichbedeutend war mit einer Risikosteigerung um 9\%. Umgerechnet heißt das: Werden 83 Patienten drei Jahre lang mit IKS gegen COPD behandelt, erleidet einer eine Fraktur, die ihm ohne die IKSBehandlung nicht widerfahren wäre.

Die relative Zunahme des Frakturrisikos um etwa $27 \%$ ist damit gegen die sinkende Gefahr von Exazerbationen der COPD abzuwägen. Die Risikoreduktion beträgt hier $20-25 \%$, die Number Needed to Treat liegt bei sechs pro Jahr. In die Therapieentscheidung sollten also das allgemeine Frakturrisiko und der Schweregrad der COPD eines Patienten einfließen.

Fazit: IKS reduzieren zwar das Exazerbationsrisiko bei COPD, erhöhen dafür aber signifikant die Gefahr, dass sich die Patienten einen Knochen brechen. Von 83 mit IKS behandelten Patienten zieht sich einer binnen drei Jahren eine zu- sätzliche Fraktur zu, andererseits wird bei sechs Patienten, die ein Jahr lang IKS erhalten, eine Exazerbation der COPD vermieden. Nutzen und Risiken der IKS müssen also abgewogen werden, wobei sowohl die generelle Frakturgefährdung eines Patienten als auch die schwere seines Lungenleidens eine Rolle spielen müssen.

Dr. Robert Bublak

Loke YK et al. Risk of fractures with inhaled corticosteroids in COPD: systematic review and meta-analysis of randomised controlled trials and observational studies. Thorax 2011; 66: 699-708

Kommentar: Exogen zugeführte Kortikosteroide sind die häufigste Ursache einer Medikamenten-induzierten Osteoporose. In vielen Fällen trägt aber auch die mit Glukokortikoiden behandelte Krankheit selbst zur Osteoporose bei, so bei Morbus Crohn, rheumatischen Erkrankungen, Kollagenosen, Transplantationen, Asthma bronchiale, malignen Lymphomen und Myelomen. Der Einsatz von Kortikosteroiden über wenige Tage verursacht selbst in sehr hoher Dosierung keinen klinisch relevanten Knochenschwund, aber bereits nach wenigen Monaten systemischer Therapie ist mit einem verstärkt einsetzenden Knochenabbau zu rechnen. Unter einer mehrjährigen Steroid-Langzeittherapie erleiden ungefähr $50 \%$ der Patienten eine manifeste Osteoporose. Die Relevanz einer lokalen Applikation von Kortikosteroiden (Cremes, Salben, Lotionen oder inhalative Darreichungsformen) auf Knochendichte und Frakturrisiko fand bisher im klinischen Alltag dagegen wenig Beachtung.

Die Metaanalyse inhalativer Kortikoide gegen COPD von Y. Loke et al. konnte statistisch belegen, dass sich auch lokal applizierte Kortikosteroide negativ auf die Knochendichte auswirken und das Frakturrisiko signifikant um $27 \%$ steigern. Wie zu erwarten, spielen dabei die Therapiedauer und und die tägliche Dosis (Anzahl der Hübe) eine wichtige Rolle. Einer Faustregel zufolge ist ein relevanter Knochenschwund bei mehr als sechs Hüben Kortikosteroid pro Tag anzunehmen. Die Metaanalyse von Loke lässt den Schluss zu, dass auch bei einer langzeitigen inhalativen Steroidapplikation eine DXA-Ausgangsmessung der Knochendichte sinnvoll ist, um das weitere Risiko abschätzen und die Effektivität einer anschließenden medikamentösen Osteoporosetherapie kontrollieren zu können. Eine weitere große Metaanalyse von Studien bei Glukokortikoid-induzierter Osteoporose aus dem Jahr 2002 zeigte, dass unter Bisphosphonaten die Knochendichte der Wirbelsäule um 4,6\% zunimmt, unter Teriparatid gar um $9,8 \%$. Dabei wurden Wirbelfrakturen um 40-70\% reduziert.

Abhängig von der aktuellen Knochenmasse bieten sich bei COPD-Patienten unter inhalativen Kortikoiden folgende Behandlungsstrategien des Knochenschwunds an: _ Normale Knochendichte oder leichte

Osteopenie (T-Score > -1,5) ohne weitere Risikofaktoren: Kalziumreiche Kost, Vitamin D (2000 I.E./Tag) und Muskeltraining, DXA-Kontrolle in halbjährlichen bis jährlichen Abständen.

_ Osteopenie oder Osteoporose bei TScore <-1,5: zusätzlich zur Basistherapie Einsatz eines stickstoffhaltigen Bisphosphonats oral oder - besser verträglich und mit höherer Compliance verbunden - intravenös (Jahresinfusion oder Vierteljahresspritze).

_ Bei manifester Osteoporose sollte alternativ eine Parathormontherapie erwogen werden.

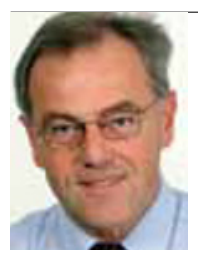

Prof. Dr. Reiner Bartl Osteoporosezentrum am Dom Kaufingerstr. 15 80331 München 